\title{
INTERPOLATIONS OF JENSEN'S INEQUALITY
}

\author{
S. S. DRAGOMIR, C. E. M. PEARCE AND J. PEČARIĆ
}

\begin{abstract}
Weighted and unweighted interpolations of general order are given for Jensen's integral inequality. Various upper-bound estimates are made for the differences between the interpolates and some convergence results derived. The results generalise and subsume a body of earlier work and employ streamlined proofs.
\end{abstract}

\section{Introduction}

A central tool in the applied literature is Jensen's weighted integral inequality, the basic form of which is as follows.

Theorem 1. Let $f, g:[a, b] \rightarrow \mathbb{R}$ be measurable and denote by $I$ the convex hull of the image of $[a, b]$ under $f$. Let $\phi: I \rightarrow R$ be convex and suppose that $g, f g$ and $(\phi \circ f) \cdot g$ are all integrable on $[a, b]$. If $g(t) \geq 0$ on $[a, b]$ and $\int_{a}^{b} g(t) d t>0$, then

$$
\phi\left(\frac{\int_{a}^{b} f(t) g(t) d t}{\int_{a}^{b} g(t) d t}\right) \leq \frac{\int_{a}^{b}(\phi \circ f)(t) g(t) d t}{\int_{a}^{b} g(t) d t}
$$

A convenient standardisation is suggested by the ubiquitous applications of Jensen's inequality in probability. If we define

$$
p(t):=g(t) / \int_{a}^{b} g(t) d t,
$$

then $p$ is nonnegative and satisfies $\int_{a}^{b} p(t) d t=1$ and so may be regarded as a probability density function on $[a, b]$. With this notation, (1.1) takes the simple form

$$
\phi\left(\int_{a}^{b} f(t) p(t) d t\right) \leq \int_{a}^{b}(\phi \circ f)(t) p(t) d t .
$$

Without loss of generality we may work with this simpler canonical form.

Received April 29, 2002.

2000 Mathematics Subject Classification. 26D15.

Key words and phrases. Convexity, Jensen's inequality, Hadamard's inequality. 
Recently Dragomir and Goh [10] derived an estimate for the difference between the two sides of a multivariate version of (1.1). In our present notation, the univariate case of their estimate is

$$
\begin{aligned}
0 \leq & \int_{a}^{b}(\phi \circ f)(t) p(t) d t-\phi\left(\int_{a}^{b} f(t) p(t) d t\right) \\
\leq & \int_{a}^{b}\left(\phi^{\prime} \circ f\right)(t) \cdot f(t) p(t) d t \\
& \quad-\int_{a}^{b}\left(\phi^{\prime} \circ f\right)(t) p(t) d t \cdot \int_{a}^{b} f(t) p(t) d t
\end{aligned}
$$

provided that all the integrals exist and $\phi$ is differentiable convex on $\mathbb{R}$.

In this paper we give some refinements of these results. For notational convenience, we introduce the $k$-variate linear integral operator

$$
I_{k}\{\cdot\}:=\int_{a}^{b} \ldots \int_{a}^{b}(\cdot) p\left(t_{1}\right) \ldots p\left(t_{k}\right) d t_{1} \ldots d t_{k} .
$$

In this notation, (1.2) now becomes

$$
\phi\left(I_{1}\{f(t)\}\right) \leq I_{1}\{(\phi \circ f)(t)\}
$$

and (1.3) reads

$$
\begin{aligned}
0 & \leq I_{1}\{(\phi \circ f)(t)\}-\phi\left(I_{1}\{f(t)\}\right) \\
& \leq I_{1}\left\{\left(\phi^{\prime} \circ f\right)(t) \cdot f(t)\right\}-I_{1}\left\{\left(\phi^{\prime} \circ f\right)(t)\right\} \cdot I_{1}\{f(t)\} .
\end{aligned}
$$

In Section 2 we interpolate (1.4), using both weighted and unweighted (that is, uniformly weighted) forms. The $k$-th order weighted and unweighted interpolates are respectively

$$
\varphi_{k}(u):=I_{k}\left\{\phi\left(\sum_{i=1}^{k} u_{i} f\left(t_{i}\right)\right)\right\}
$$

and

$$
\varphi_{k}:=I_{k}\left\{\phi\left(\frac{1}{k} \sum_{i=1}^{k} f\left(t_{i}\right)\right)\right\} .
$$

Here $u=\left(u_{1}, \ldots, u_{k}\right)$ is a set of probability weights, that is, each $u_{i} \geq 0$ and $\sum_{i=1}^{k} u_{i}=1$, and it is envisaged that $k$ is a fixed positive integer. When we wish to vary the order $k$ the extended notation $u^{(k)}=\left(u_{1, k}, \ldots, u_{k, k}\right)$ will be used.

The basic result is Theorem 2, which generalises a number of known results. We shall see that the $k$-th order weighted interpolate $\varphi_{k}(u)$ is minimised by the unweighted interpolate $\varphi_{k}$, that is, when each $u_{i}=1 / k$. In Section 3 we give upper bounds for the difference between the first and third terms in (2.1) below. By virtue of the noted minimisation result, our estimates include as a special case an upper bound for the difference 
between the first and second terms in (2.1). A convergence theorem is established for the difference with $k \rightarrow \infty$.

In Section 4 we treat the sequence $\left(\varphi_{k}\left(u^{(k)}\right)-\varphi_{k}\right)_{k \geq 1}$. Some results for the sequence $\left(\varphi_{k}-\varphi_{k+1}\right)_{k \geq 1}$ are deduced in Section 5 . We conclude in Section 6 with some remarks on applications to Hadamard's inequalities.

Our arguments exploit the standardisation of $p$ being a probability density. Suppose $Y_{1}, \ldots, Y_{k}$ are independent random variables with common density function $p$ and define $X_{1}, \ldots, X_{k}$ by $X_{i}=f\left(Y_{i}\right)(i=1, \ldots, k)$. We shall also write $X, Y$ for a generic pair $X_{i}, Y_{i}$. Then $I_{k}$ is simply the expectation operator with respect to the minimal completed sigma field $\mathbf{F}_{k}$ generated by $Y_{1}, \ldots, Y_{k}$. Denoting the mean of $X_{1}$ by $E\left(X_{1}\right)$, as is customary, we then have $E\left(X_{1}\right)=I_{1}\left\{f\left(t_{1}\right)\right\}$. Since $\mathbf{F}_{1}$ is a sub sigma field of $\mathbf{F}_{k}$, we have also $E\left(X_{1}\right)=I_{k}\left\{f\left(t_{1}\right)\right\}$. We may now express (1.2), (1.5) slightly more succinctly and considerably more evocatively as respectively

$$
\phi(E(X)) \leq E(\phi(X))
$$

and

$$
0 \leq E(\phi(X))-\phi(E(X)) \leq E\left(X \phi^{\prime}(X)\right)-E\left(\phi^{\prime}(X)\right) E(X) .
$$

We shall lean heavily on this probabilistic formulation both for compact notation within our proofs and for streamlining the algebra involved in them. The assumptions of Theorem 1 are presumed throughout without further comment and with the standardision that $g$ is replaced by a probability density function $p$. A number of useful bounds arise via the Cauchy-Schwarz inequality. In each such connection we shall assume in addition without further comment that $f^{2}$ is integrable, and introduce

$$
\sigma:=\left[I_{1}\left\{f^{2}(t)\right\}-\left(I_{1}\{f(t)\}\right)^{2}\right]^{1 / 2} .
$$

Probabilistically this states that

$$
\sigma^{2}=E\left(X^{2}\right)-[E(X)]^{2}=: \operatorname{var}(X),
$$

the variance of $X$. The basic probabilistic results we shall invoke are that $E\left(U^{2}\right)=\operatorname{var}(U)$ when $E(U)=0$ and that for independent random variables $X_{1}, \ldots, X_{k}$ and constants $u_{1}, \ldots, u_{k}$, we have

$$
\operatorname{var}\left(\sum_{i=1}^{k} u_{i} X_{i}\right)=\sum_{i=1}^{k} u_{i}^{2} \operatorname{var}\left(X_{i}\right)
$$

For notation convenience, it will be convenient to introduce into our discussion the auxiliary random variables

$$
Z_{1}=Z_{1, k}:=\sum_{i=1}^{k} u_{i} X_{i}
$$

and

$$
W_{k}:=\frac{1}{k} \sum_{i=1}^{k} X_{i} \text {. }
$$


It is immediate that $E\left(Z_{1}\right)=E\left(W_{k}\right)=E(X)$ and that $\varphi_{k}(u)=E\left(\phi\left(Z_{1}\right)\right)$ and $\varphi_{k}=$ $E\left(\phi\left(W_{k}\right)\right)$.

\section{Basic Results}

Our first result relates expectations involving weighted and unweighted interpolates and refines (1.1).

Theorem 2. For each $k \geq 1$ and set of probability weights $u^{(k)}$, we have

$$
\phi\left(I_{1}\{f(t)\}\right) \leq \varphi_{k} \leq \varphi_{k}\left(u^{(k)}\right) \leq I_{1}\{(\phi \circ f)(t)\} .
$$

Proof. In probabilistic terms, the result to be proved is that

$$
\phi(E(X)) \leq E\left(\phi\left(W_{k}\right)\right) \leq E\left(\phi\left(Z_{1}\right)\right) \leq E(\phi(X)) .
$$

By Jensen's integral inequality we have

$$
E\left\{\phi\left(W_{k}\right)\right\} \geq \phi\left(E\left\{\left(W_{k}\right)\right\}\right)=\phi(E(X)),
$$

the first inequality in the enunciation.

For fixed $k$ put $X_{i+k}:=X_{i}$ and for $1 \leq j \leq k$ define

$$
Z_{j}:=\sum_{i=1}^{k} u_{i} X_{i+j-1}
$$

which is consistent with the definition of $Z_{1}$. Then $E\left(Z_{j}\right)=E(X)$ and $E\left(\phi\left(Z_{j}\right)\right)=$ $E\left(\phi\left(Z_{1}\right)\right)$.

By Jensen's discrete inequality we have

$$
\phi\left(\frac{1}{k} \sum_{i=1}^{k} Z_{i}\right) \leq \frac{1}{k} \sum_{i=1}^{k} \phi\left(Z_{i}\right),
$$

and since $\sum_{i=1}^{k} Z_{i}=k W_{k}$, we derive

$$
\phi\left(W_{k}\right) \leq \frac{1}{k} \sum_{j=1}^{k} \phi\left(Z_{j}\right) .
$$

Taking expectations provides

$$
E\left\{\phi\left(W_{k}\right)\right\} \leq \frac{1}{k} E\left\{\sum_{j=1}^{k} \phi\left(Z_{j}\right)\right\}=E\left\{\phi\left(Z_{1}\right)\right\} .
$$

This gives the second inequality in the enunciation. 
Finally, by Jensen's discrete inequality again, we have

$$
\phi\left(Z_{1}\right) \leq \sum_{i=1}^{k} u_{i} \phi\left(X_{i}\right) .
$$

Taking expectations provides the final desired inequality.

If we choose $u_{i, k+1}=1 / k$ for $1 \leq i \leq k$ and $u_{k+1, k+1}=0$, then $\varphi_{k+1}(u)$ becomes $\varphi_{k}$. Thus we have $\varphi_{k+1} \leq \varphi_{k}$ and so $\left(\varphi_{k}\right)_{k \geq 1}$ is a nonincreasing sequence. We have also $\varphi_{1}=E(\phi(X))$, of course.

The choices $f(t):=t$ and $p(t):=1 /(b-a)$ provide the following interpolation of the Hadamard integral inequalities, which we exhibit in extenso.

Corollary 1. Suppose $\phi$ is convex on $[a, b]$ and that $u_{i}(1 \leq i \leq k)$ is a set of probability weights. Then

$$
\begin{aligned}
\phi\left(\frac{a+b}{2}\right) & \leq \frac{1}{(b-a)^{k}} \int_{a}^{b} \ldots \int_{a}^{b} \phi\left(\frac{1}{k} \sum_{i=1}^{k} t_{i}\right) d t_{1} \ldots d t_{k} \\
& \leq \frac{1}{(b-a)^{k}} \int_{a}^{b} \ldots \int_{a}^{b} \phi\left(\sum_{i=1}^{k} u_{i} t_{i}\right) d t_{1} \ldots d t_{k} \\
& \leq \frac{1}{b-a} \int_{a}^{b} \phi(t) d t .
\end{aligned}
$$

This subsumes several known results: the first inequality was proved in [11], the second in [8] and the last in [4]. We pick up these threads again in Section 6.

\section{Bounds for the Difference $\varphi_{k}(u)-\phi\left(I_{1}\{f(t)\}\right)$}

Theorem 3. Denote by $\phi_{+}^{\prime}$ the right derivative of $\phi$ on the interior I of $I$. Then

$$
\begin{aligned}
0 & \leq \varphi_{k}(u)-\phi\left(I_{1}\{f(t)\}\right) \\
& \leq I_{k}\left\{\phi_{+}^{\prime}\left(\sum_{i=1}^{k} u_{i} f\left(t_{i}\right)\right) \sum_{j=1}^{k} u_{j} f\left(t_{j}\right)\right\}-I_{1}\{f(t)\} \cdot I_{k}\left\{\phi_{+}^{\prime}\left(\sum_{i=1}^{k} u_{i} f\left(t_{i}\right)\right)\right\} .
\end{aligned}
$$

Proof. We already have the first inequality and wish to prove the second. We may express (3.1) probabilistically as

$$
0 \leq E\left\{\phi Z_{1}\right\}-E(X) \leq E\left\{Z_{1} \phi_{+}^{\prime}\right\}-E(X) \cdot E\left\{\phi_{+}^{\prime}\right\} .
$$

Since $\phi$ is convex on $I$,

$$
\phi(x)-\phi(y) \geq \phi_{+}^{\prime}(y)(x-y) \text { for all } x, y \in \stackrel{\circ}{\mathrm{I}}
$$


and $\phi_{+}^{\prime}(\cdot)$ is nonnegative on I. Taking $x=E(X)$ and $y=Z_{1}$, we deduce that

$$
\phi(E(X))-\phi\left(Z_{1}\right) \geq \phi_{+}^{\prime}\left(Z_{1}\right)\left[E(X)-Z_{1}\right] .
$$

Taking expectations yields

$$
E\left\{\phi\left(Z_{1}\right)\right\}-\phi\left(E\left(Z_{1}\right)\right) \leq E\left\{Z_{1} \phi_{+}^{\prime}\left(Z_{1}\right)\right\}-E\left\{Z_{1}\right\} \cdot E\left\{\phi_{+}^{\prime}\left(Z_{1}\right)\right\},
$$

whence we have the desired result.

When each $u_{i}=1 / k$, we may exploit symmetry in $j$ of the summand in (3.1) to simplify the conclusion of the last theorem to

$$
\begin{aligned}
0 & \leq \varphi_{k}(u)-\phi\left(I_{1}\{f(t)\}\right) \\
& \leq I_{k}\left\{f\left(t_{1}\right) \phi_{+}^{\prime}\left(\frac{1}{k} \sum_{i=1}^{k} f\left(t_{i}\right)\right)\right\}-I_{1}\{f(t)\} \cdot I_{k}\left\{\phi_{+}^{\prime}\left(\frac{1}{k} \sum_{i=1}^{k} f\left(t_{i}\right)\right)\right\} .
\end{aligned}
$$

The previous theorem may be extended as follows.

Theorem 4. For $k \geq 1$ we have

$$
\begin{aligned}
0 & \leq I_{k}\left\{\phi_{+}^{\prime}\left(\sum_{i=1}^{k} u_{i} f\left(t_{i}\right)\right) \sum_{j=1}^{k} u_{j} f\left(t_{j}\right)\right\}-I_{1}\{f(t)\} \cdot I_{k}\left\{\phi_{+}^{\prime}\left(\sum_{i=1}^{k} u_{i} f\left(t_{i}\right)\right)\right\} \\
& \leq \sigma \sqrt{\sum_{j=1}^{k} u_{j}^{2}}\left[I_{k}\left\{\left[\phi_{+}^{\prime}\left(\sum_{i=1}^{k} u_{i} f\left(t_{i}\right)\right)\right]^{2}\right\}\right]^{1 / 2} .
\end{aligned}
$$

Proof. Since $E(X)=E\left(Z_{1}\right)$, the middle term in (3.3) can be cast probabilistically as

$$
E\left\{\phi_{+}^{\prime}\left(Z_{1}\right) \times\left[Z_{1}-E\left(Z_{1}\right)\right]\right\},
$$

which by the Cauchy-Schwarz inequality is less than or equal to

$$
\left\{E\left\{\left[\phi_{+}^{\prime}\left(Z_{1}\right)\right]^{2}\right\}\right\}^{1 / 2}\left\{E\left\{\left[Z_{1}-E\left\{Z_{1}\right\}\right]^{2}\right\}\right\}^{1 / 2} .
$$

Further $E\left[Z_{1}-E\left(Z_{1}\right)\right]=0$, so we have

$$
E\left\{\left[Z_{1}-E\left(Z_{1}\right)\right]^{2}\right\}=\operatorname{var}\left(Z_{1}\right)=\sum_{i=1}^{k} u_{i}^{2} \operatorname{var}\left(X_{i}\right)=\sigma^{2} \sum_{i=1}^{k} u_{i}^{2},
$$

and the desired result follows. 
As with the previous theorem, (3.3) simplifies when $u_{i}=1 / k$ for each $i$, becoming

$$
\begin{aligned}
0 & \leq I_{k}\left\{\phi_{+}^{\prime}\left(\frac{1}{k} \sum_{i=1}^{k} f\left(t_{i}\right)\right) f\left(t_{1}\right)\right\}-I_{1}\left\{f(t\} \cdot I_{k}\left\{\phi_{+}^{\prime}\left(\frac{1}{k} \sum_{i=1}^{k} f\left(t_{i}\right)\right)\right\}\right. \\
& \leq \sigma k^{-1 / 2}\left[I_{k}\left\{\left[\phi_{+}^{\prime}\left(\frac{1}{k} \sum_{i=1}^{k} f\left(t_{i}\right)\right)\right]^{2}\right\}\right]^{1 / 2} .
\end{aligned}
$$

for all $k \geq 1$.

\section{Corollary 2. Suppose that}

$$
M:=\sup _{x \in I}\left|\phi_{+}^{\prime}(x)\right|<\infty
$$

and

$$
\sum_{j=1}^{k} u_{j, k}^{2} \rightarrow 0 \text { as } k \rightarrow \infty
$$

Then

$$
\varphi_{k}\left(u^{(k)}\right) \rightarrow \phi\left(I_{1}\{f(t)\}\right) \text { as } k \rightarrow \infty .
$$

We note that the second assumption is automatically satisfied in the particular case $u_{j, k}=1 / k$ for $1 \leq j \leq k$.

The conclusion of the corollary may be expressed probabilistically as

$$
E\left\{Z_{1, k}\right\} \rightarrow \phi(E(X)) \text { as } k \rightarrow \infty .
$$

\section{Bounds for $\varphi_{k}(u)-\varphi_{k}$}

The difference between the outermost terms in (2.1) can be used to provide a crude upper bound for $\varphi_{k}(u)-\varphi_{k}$. Here we provide tighter bounds.

Theorem 5. For $k \geq 1$ we have

$$
\begin{aligned}
0 & \leq \varphi_{k}(u)-\varphi_{k} \\
& \leq I_{k}\left\{\phi_{+}^{\prime}\left(\sum_{i=1}^{k} u_{i} f\left(t_{i}\right)\right) \sum_{j=1}^{k} u_{j} f\left(t_{j}\right)\right\}-I_{k}\left\{\phi_{+}^{\prime}\left(\sum_{i=1}^{k} u_{i} f\left(t_{i}\right)\right) \frac{1}{k} \sum_{j=1}^{k} f\left(t_{j}\right)\right\} .
\end{aligned}
$$

Proof. By the convexity of $\phi$

$$
\phi\left(W_{k}\right)-\phi\left(Z_{1}\right) \geq \phi_{+}^{\prime}\left(Z_{1}\right)\left(W_{k}-Z_{1}\right) .
$$

Taking expectations provides

$$
E\left(\phi\left(Z_{1}\right)\right)-E\left(\phi\left(W_{k}\right)\right) \leq E\left\{Z_{1} \phi_{+}^{\prime}\left(Z_{1}\right)\right\}-E\left\{W_{k} \phi_{+}^{\prime}\left(Z_{1}\right)\right\},
$$


which is the desired result in probabilistic form.

The estimate is continued by the next theorem.

Theorem 6. For each $k \geq 1$,

$$
\begin{gathered}
I_{k}\left\{\phi_{+}^{\prime}\left(\sum_{i=1}^{k} u_{i} f\left(t_{i}\right)\right) \sum_{j=1}^{k} u_{j} f\left(t_{j}\right)\right\}-I_{k}\left\{\phi_{+}^{\prime}\left(\sum_{i=1}^{k} u_{i} f\left(t_{i}\right)\right) \frac{1}{k} \sum_{j=1}^{k} f\left(t_{j}\right)\right\} \\
\leq \sigma \sqrt{\sum_{i=1}^{k}\left(u_{i}-1 / k\right)^{2}} \times\left[I_{k}\left\{\left[\phi_{+}^{\prime}\left(\sum_{j=1}^{k} u_{j} f\left(t_{j}\right)\right)\right]^{2}\right\}\right]^{1 / 2} .
\end{gathered}
$$

Proof. The left-hand side of this inequality is

$$
E\left\{\phi_{+}^{\prime}\left(Z_{1}\right) \times\left(Z_{1}-W_{k}\right)\right\},
$$

which by the Cauchy-Schwarz inequality is less than or equal to

$$
\left(E\left\{\left[\phi_{+}^{\prime}\left(Z_{1}\right)\right]^{2}\right\}\right)^{1 / 2} \times\left(E\left\{\left[Z_{1}-W_{k}\right]^{2}\right\}\right)^{\frac{1}{2}} .
$$

Since $E\left(Z_{1}-W_{k}\right)=0$, we may compute the second term in parentheses as

$$
\operatorname{var}\left\{\sum_{j=1}^{k}\left(u_{j}-\frac{1}{k}\right) X_{j}\right\}=\sum_{j=1}^{k}\left(u_{j}-\frac{1}{k}\right)^{2} \operatorname{var}\left(X_{j}\right)=\sigma^{2} \sum_{j=1}^{k}\left(u_{j}-\frac{1}{k}\right)^{2},
$$

from which we deduce the desired estimate.

Corollary 3. If (3.4) applies, then

$$
0 \leq \varphi_{k}(u)-\varphi_{k} \leq M \sigma\left[\sum_{i=1}^{k}\left(u_{i}-\frac{1}{k}\right)^{2}\right] .
$$

It follows that subject to (3.4), a sufficient condition for

$$
\lim _{k \rightarrow \infty} \varphi_{k}(u)=\phi\left(I_{1}\{f(t)\}\right)
$$

is that

$$
\lim _{k \rightarrow \infty} \sum_{i=1}^{k}\left(u_{i, k}-1 / k\right)^{2}=0 .
$$

By virtue of the relation $\sum_{i=1}^{k} u_{i, k}=1$, this is the same condition as (3.5). 


\section{Upper Bounds for $\varphi_{k}-\varphi_{k+1}$}

From (2.2), we have

$$
\phi(E(X)) \leq \varphi_{k+1} \leq \varphi_{k} \leq \ldots \leq E(\phi(X))
$$

for $k \geq 1$, so that the difference $\varphi_{k}-\varphi_{k+1}$ is nonnegative and can be ascribed a uniform upper bound $E(\phi(X))-\phi(E(X))$ which is independent of $k$. The next theorem refines this to a tighter and $k$-dependent bound.

Theorem 7. For each $k \geq 1$,

$$
\begin{aligned}
0 & \leq \varphi_{k}-\varphi_{k+1} \\
& \leq \frac{1}{k+1}\left[I_{k}\left\{\phi_{+}^{\prime}\left(\frac{1}{k} \sum_{i=1}^{k} f\left(t_{i}\right)\right) f\left(t_{1}\right)\right\}-I_{k}\left\{\phi_{+}^{\prime}\left(\frac{1}{k} \sum_{i=1}^{k} f\left(t_{i}\right)\right)\right\} I_{1}\{f(t)\}\right] .
\end{aligned}
$$

Proof. As $\phi$ is convex,

$$
\begin{aligned}
\phi\left(\frac{1}{k+1} \sum_{i=1}^{k+1} X_{i}\right)-\phi\left(\frac{1}{k} \sum_{i=1}^{k} X_{i}\right) & \geq \phi_{+}^{\prime}\left(\frac{1}{k} \sum_{i=1}^{k} X_{i}\right)\left(\frac{1}{k+1} \sum_{j=1}^{k+1} X_{j}-\frac{1}{k} \sum_{j=1}^{k} X_{j}\right) \\
& =\phi_{+}^{\prime}\left(\frac{1}{k} \sum_{i=1}^{k} X_{i}\right)\left[\frac{X_{k+1}}{k+1}-\frac{1}{k(k+1)} \sum_{j=1}^{k} X_{j}\right]
\end{aligned}
$$

for all $k \geq 1$.

Taking expectations provides

$$
\begin{aligned}
\varphi_{k+1}-\varphi_{k} \geq \frac{1}{k+1}\left[E\left\{\phi_{+}^{\prime}\left(\frac{1}{k} \sum_{i=1}^{k} X_{i}\right)\right\} E(X)-E\left\{\phi_{+}^{\prime}\left(\frac{1}{k} \sum_{i=1}^{k} X_{i}\right) \frac{1}{k} \sum_{j=1}^{k} X_{j}\right\}\right] \\
=\frac{1}{k+1}\left[E\left\{\phi_{+}^{\prime}\left(\frac{1}{k} \sum_{i=1}^{k} X_{i}\right)\right\} E(X)-E\left\{X_{1} \phi_{+}^{\prime}\left(\frac{1}{k} \sum_{i=1}^{k} X_{i}\right)\right\}\right],
\end{aligned}
$$

where symmetry has been coupled with a change of variables to provide the last step.

The above result is continued by the following one.

Theorem 8. For each $k \geq 1$ we have

$$
\begin{gathered}
\frac{1}{k+1}\left[I_{k}\left\{\phi_{+}^{\prime}\left(\frac{1}{k} \sum_{i=1}^{k} f\left(t_{i}\right)\right) f\left(t_{1}\right)\right\}-I_{k}\left\{\phi_{+}^{\prime}\left(\frac{1}{k} \sum_{i=1}^{k} f\left(t_{i}\right)\right)\right\} I_{1}\{f(t)\}\right] \\
\leq \frac{\sigma}{\sqrt{k(k+1)}}\left[I_{k}\left\{\left[\phi_{+}^{\prime}\left(\frac{1}{k} \sum_{i=1}^{k} f\left(t_{i}\right)\right)\right]^{2}\right\}\right]^{1 / 2} .
\end{gathered}
$$


Proof. The left-hand side can be expressed as

$$
\frac{1}{k+1} E\left\{\phi_{+}^{\prime}\left(\frac{1}{k} \sum_{i=1}^{k} X_{i}\right)\left[X_{k+1}-\frac{1}{k} \sum_{j=1}^{k} X_{j}\right]\right\}
$$

which by the Cauchy-Schwarz inequality is less than or equal to

$$
\left(E\left\{\left[\phi_{+}^{\prime}\left(\frac{1}{k} \sum_{i=1}^{k} X_{i}\right)\right]^{2}\right\}\right)^{1 / 2} \times\left(E\left\{\left[X_{k+1}-\frac{1}{k} \sum_{i=1}^{k} X_{i}\right]^{2}\right\}\right)^{1 / 2} .
$$

Since $E\left(X_{k+1}-(1 / k) \sum_{i=1}^{k} X_{i}\right)=0$, the expression within the second pair of parentheses is

$$
\operatorname{var}\left(X_{k+1}-\frac{1}{k} \sum_{i=1}^{k} X_{i}\right)=\operatorname{var}\left(X_{k+1}\right)+\frac{1}{k^{2}} \sum_{i=1}^{k} \operatorname{var}\left(X_{i}\right)=\frac{(k+1) \sigma^{2}}{k},
$$

from which we deduce the desired result.

Finally we have the following corollary.

Corollary 4. If (3.4) holds, then for all $\alpha \in[0,1)$ we have

$$
\lim _{n \rightarrow \infty}\left(\varphi_{n}-\varphi_{n+1}\right) n^{\alpha}=0
$$

Proof. By the two preceding theorems,

$$
0 \leq \varphi_{n}-\varphi_{n+1} \leq \frac{M \sigma}{\sqrt{n(n+1)}}
$$

for all $n \geq 1$, whence the result.

\section{Applications to Hadamard's Inequalities}

We conclude by resuming from Corollary 1 and the observations made there. Hadamard's inequality states that if $\phi: I \rightarrow \mathbb{R}$ is convex on the interval $I=[a, b]$ of real numbers, then

$$
\phi\left(\frac{a+b}{2}\right) \leq \frac{1}{b-a} \int_{a}^{b} \phi(x) d x \leq \frac{\phi(a)+\phi(b)}{2} .
$$

Denote by

$$
J_{k}\{\cdot\}:=\frac{1}{(b-a)^{k}} \int_{a}^{b} \ldots \int_{a}^{b}(\cdot) d x_{1} \ldots d x_{k}
$$


the special case of $I_{k}$ when $p(x):=1 /(b-a)$ on $[a, b]$. Dragomir, Pečarić and Sándor [11] have interpolated the first inequality in (6.1) as

$$
\phi\left(\frac{a+b}{2}\right) \leq J_{k+1}\left\{\phi\left(\frac{1}{k+1} \sum_{i=1}^{k+1} x_{i}\right)\right\} \leq J_{k}\left\{\phi\left(\frac{1}{k} \sum_{i=1}^{k} x_{i}\right)\right\} \leq \ldots \leq J_{1}\{\phi(x)\}
$$

for all $k \geq 1$. This is a particular case of (5.1).

Dragomir [4] has also established a weighted interpolation, in our notation

$$
\phi\left(\frac{a+b}{2}\right) \leq J_{k}\left\{\phi\left(\sum_{i=1} u_{i} x_{i}\right)\right\} \leq J_{1}\{\phi(x)\}
$$

of Hadamard's first inequality. This was subsequently improved by Dragomir and Buşe [8] who proved inter alia that

$$
J_{k}\left\{\phi\left(\frac{1}{k} \sum_{i=1}^{k} x_{i}\right)\right\} \leq J_{k}\left\{\phi\left(\sum_{i=1}^{k} u_{i} x_{i}\right)\right\} .
$$

This is Theorem 2 with $f(x):=x$ (and so $X_{i}=Y_{i}$ ).

From Corollary 2 we can obtain the following result which was derived by a different argument in [9].

Suppose $\phi: I \rightarrow \mathbb{R}$ is convex, (3.4) holds and that

$$
\lim _{n \rightarrow \infty} \frac{\sum_{i=1}^{n} v_{i}^{2}}{\left(\sum_{i=1}^{n} v_{i}\right)^{2}}=0 .
$$

Then if $V_{n}:=\sum_{i=1}^{n} v_{i}>0$, we have

$$
\lim _{n \rightarrow \infty} J_{n}\left\{\phi\left(\sum_{i=1}^{n} v_{i} x_{i} / V_{n}\right)\right\}=\phi\left(\frac{a+b}{2}\right) .
$$

Write $h_{n}, h_{n}(u)$ respectively for $\varphi_{n}, \varphi_{n}(u)$ in the case $p(x)=1 /(b-a)$ on $[a, b]$. We have the following.

Proposition 1. Let $\phi: I \rightarrow \mathbb{R}$ be convex and suppose (3.4) holds. Then for all $a, b \in I$ with $a<b$, we have

$$
0 \leq h_{n}-h_{n+1} \leq \frac{M(b-a)}{2 \sqrt{3} \sqrt{n(n+1)}}
$$

for all positive integers $n$.

Proof. The result is (5.3) with

$$
\sigma^{2}=\frac{\int_{a}^{b} t^{2} d t}{b-a}-\left(\frac{\int_{a}^{b} t d t}{b-a}\right)^{2}=\frac{(b-a)^{2}}{12} .
$$


The consequence

$$
\lim _{n \rightarrow \infty}\left[n^{\alpha}\left(h_{n}-h_{n+1}\right)\right]=0 \text { for } \alpha \in[0,1)
$$

is an improvement on the results of [7].

The weighted case is embodied in the following proposition.

Proposition 2. With the assumptions of Proposition 1,

$$
0 \leq h_{n}(u)-h_{n} \leq \frac{M(b-a)}{2 \sqrt{3}}\left[\sum_{i=1}^{n}\left(u_{i}-1 / n\right)^{2}\right]^{1 / 2}
$$

for all $n \geq 1$.

For other results connected with Hadamard's inequality see [1]-[9], where further references are given.

\section{Acknowledgement}

This work was done during a visit of the last-named author to The University of Adelaide.

\section{References}

[1] S. S. Dragomir, Two refinements of Hadamard's inequalities, Coll. of Sci. Pap. of the Fac. of Sci., Kragujevac 11(1990), 23-26.

[2] S. S. Dragomir, A mapping in connection with Hadamard's inequalities, Ann. Östr. Akad. Wiss. Math.-Maths 128(1991), 17-20.

[3] S. S. Dragomir, Two mappings in connection to Hadamard's inequality, J. Math. Anal. Appl. 167(1992), 45-56.

[4] S. S. Dragomir, On Hadamard's inequality for convex functions, Mat. Balkanica 6(1992), 215-222.

[5] S. S. Dragomir, A refinement of Hadamard's inequality for isotonic linear functionals, Tamkang J. Math. 24(1993), 101-106.

[6] S. S. Dragomir, Some remarks on Hadamard's inequality for convex functions, Extracta Math. 9(1994), 89-94.

[7] S. S. Dragomir, D. Barbu and C. Buşe, A probabilistic argument for the convergence of some sequences associated with Hadamard's inequality, Studia Univ. Babes-Bolyai Math. 38 (1993), 29-33.

[8] S. S. Dragomir and C. Buşe, Refinements of Hadamard's inequality for multiple integrals, Utilitas Math. 47(1995), 193-195.

[9] S. S. Dragomir, C. Buşe and D. Barbu, The convergence of some sequences connected to Hadamard's inequality, Demonstratio Math. 29(1996), 53-59.

[10] S. S. Dragomir and C. J. Goh, A counterpart of Jensen's continuous inequality and applications in information theory, RGMIA http://rgmia.vu.edu.au/Jensen.html 
[11] S. S. Dragomir, J. Pečarić and J. Sándor, A note on the Jensen-Hadamard inequality, Anal. Num. Theor. Approx. 19(1990), 21-28.

[12] J. Pečarić and S. S. Dragomir, A refinement of Jensen's inequality and applications, Studia Univ. Babes-Bolyai 34(1989), 15-19.

School of Communications and Informatics, Victoria University of Technology, PO Box 14428, Melbourne City, MC 8001 Australia.

E-mail: cpearce@maths.adelaide.edu.au

Applied Mathematics Department, Adelaide University, Adelaide, SA 5005, Australia.

E-mail: Sever.Dragomir@vu.edu.au

Faculty of Textile Technology, University of Zagreb, Pierottijeva 6, 10000 Zagreb, Croatia.

E-mail: pecaric@mahazu.hazu.hr 